\title{
Dural Arteriovenous Fistula within Superior Sagittal Sinus Wall with Direct Cortical Venous Drainage: A Systematic Literature Review
}

\author{
Rajendra Chavan ${ }^{1}{ }^{1}$ Shreya Sethi ${ }^{1}$ Harsha Sahu $^{1}$ Neeraj Rao ${ }^{1}$ Shivani Agarwal ${ }^{1}$ \\ ${ }^{1}$ Department of Radiology, Jehangir Hospital, Pune, Maharashtra, \\ Address for correspondence Rajendra Chavan, DM, Department of \\ Radiology, Jehangir Hospital, 32, Sassoon Road, Pune, Maharashtra, \\ 411001, India (e-mail: dr_rajendrachavan@hotmail.com). \\ J Clin Interv Radiol ISVIR 2022;6:119-130.
}

\begin{abstract}
Keywords

- Type III/IV dural arteriovenous fistula on Superior sagittal sinus

- Venous ectasia/aneurysm with early rebleed

- Endovascular treatment

Dural arteriovenous fistulas (DAVFs) located within superior sagittal sinus (SSS) wall with direct cortical venous drainage are rare. They are also known as variant DAVF (VDAVF) and form a special subgroup of DAVFs. Their chance of presenting with aggressive features is high compared with transverse sigmoid sinus fistula. They drain directly into cortical veins (Borden type 3, Cognard type III and IV). A systematic English literature review of SSS vDAVF was made. Systematic literature review revealed a total of 31 published cases. These were commonly seen in male population, (24 males, $77.41 \%, 24 / 31)$. Average age of patients was 54 years. A total of 24 patients $(77.41 \%$, 24/31) had aggressive clinical presentations with 13 patients $(41.93 \%, 13 / 31)$ having intracranial hemorrhages (ICH). Two patients had rebleeding $(15.38 \%, 2 / 13)$. Middle portion of SSS was commonly involved (15 cases, 75\%). A total of 25 (96.15\%, 25/26) cases had patent SSS. Most of the fistulas were idiopathic $(65.38 \%, 17 / 26)$, with trauma being a frequent etiological factor $(26.92 \%, 7 / 26)$. Venous ectasia was seen in 19 patients $(59.37 \%, 19 / 32)$. Middle meningeal arterial (MMA) supply was seen in all patients $(100 \%, 26 / 26)$, with bilateral MMA supply in 21 cases $(80.76 \%)$, and unilateral in 5 cases (19.23\%). Twenty patients $(62.50 \%, 20 / 32)$ received only endovascular treatment (EVT), while four patients had EVT followed by surgery $(12.5 \%, 4 / 32)$. Transarterial route via MMA was the preferred treatment option (79.16\%). Complete obliteration of fistulas was noted in all cases (100\%, 30/30). No immediate complication was noted after EVT. As much as $92.30 \%$ patients showed good recovery. Thus, SSS VDAVF forms a special subgroup of DAVF, with aggressive presentation, and warrants urgent treatment. EVT is effective treatment option and can produce complete obliteration.
\end{abstract}

published online July 9,2021
DOI https://doi.org/ $10.1055 / \mathrm{s}-0041-1731594$. ISSN 2457-0214.
(C) 2021. Indian Society of Vascular and Interventional Radiology. All rights reserved.

This is an open access article published by Thieme under the terms of the Creative Commons Attribution-NonDerivative-NonCommercial-License, permitting copying and reproduction so long as the original work is given appropriate credit. Contents may not be used for commercial purposes, or adapted, remixed, transformed or built upon. (https://creativecommons.org/ licenses/by-nc-nd/4.0/)

Thieme Medical and Scientific Publishers Pvt. Ltd., A-12, 2nd Floor, Sector 2, Noida-201301 UP, India 


\section{Introduction}

Dural arteriovenous fistulas (DAVFs) are acquired lesions and constitute approximately 10 to $15 \%$ of all intracranial arteriovenous malformations (AVMs). ${ }^{1}$ They can develop spontaneously, after trauma, or after sinus thrombosis. DAVFs can occur at any location. Involvement of the superior sagittal sinus (SSS) is rare. ${ }^{2}$ DAVFs which are located in the wall of SSS with direct cortical venous drainage ([CVD], Borden type 3) are considered high-grade fistulas. ${ }^{3}$ These can be Cognard type III or IV, depending on associated venous ectasia. ${ }^{4}$ These are also labeled as variant SSS DAVFs (vSSS DAVF). Most of the time, SSS is seen patent in such vDAVF cases. This form is considered as an aggressive subtype of DAVFs. ${ }^{5}$ The incidence of intracranial hemorrhage (ICH) is reported in up to $40 \%$ of these patients, the highest in any location. ${ }^{4}$ Complexity of the draining cortical vein like venous ectasia/aneurysm, outflow restrictions, and long venous course results in higher incidence of ICH. ${ }^{6}$ Clinicians face unique problems in diagnosing and treating these DAVFs, due to their midline location, multiple arterial feeders coming from external carotid artery (ECA)/internal carotid artery (ICA)/vertebral arteries, critical venous anatomy, and adjacent eloquent brain areas. ${ }^{7}$ Various treatment options like transarterial embolization (TAE), transvenous embolization (TVE), surgical ligation, radiosurgery, or combination of these, are available. ${ }^{8,9}$ We report a case of type IV DAVF which had rebled within a few hours due to rerupture of a venous aneurysm. We reviewed all available data on such DAVFs to discuss important aspects of this rare but important intracranial vascular pathology.

\section{Illustrative Case}

A 40-year-old patient was brought to our emergency department with acute onset right-sided weakness and severe headache. He was conscious, had neck rigidity and found to have grade $0 / 5$ power in the right upper and lower limbs. His blood pressure was $124 / 82 \mathrm{~mm} \mathrm{Hg}$. He had past history of road traffic accident 4 years ago, when he sustained head injury; however, detailed records were not available.
The patients' detailed MRI examination ( - Fig. 1A, B) revealed hyperacute left parietal hematoma measuring 3.8 $\times 2.6 \times 3.5 \mathrm{~cm}$ (approximate volume $18 \mathrm{~mL}$ ). Two prominent cortical veins were seen traversing through overlying sulcal spaces on T2-weighted (T2W) images. One of these veins revealed balloon-like outpouching, lying in close proximity to the hyperacute hematoma. MR venography (MRV) revealed patent SSS with normal caliber. Few prominent veins were seen along frontoparietal convexities and scalp region. He had generalized tonic-clonic convulsion (GTC) in the hospital, became unconscious, and was intubated. CT brain study ( - Fig. 1C, D) revealed increase in the size of hematoma, now measuring approximately $6.5 \times 4.5 \times 5.4 \mathrm{~cm}$ (approximate volume $77 \mathrm{~mL}$ ). Blood was also seen in all ventricles. He also had subfalcine and uncal herniations. He was rushed to emergency left craniectomy surgery, in order to help reduce intracranial pressure (ICP).

Postcraniectomy, patient's right ECA angiograms revealed DAVF, lying within the wall of SSS. It was supplied by right middle meningeal artery (MMA) and superficial temporal artery (STA) (-Fig. 2A, B). Since he had undergone left frontoparietal craniotomy with intraoperative occlusion of left MMA, the fistula was not opacified on left ECA angiograms. This DAVF, lying within the wall of SSS wall, was drained directly into a dilated cortical vein which was, in turn, draining into two tributaries; the anterior tributary showed multiple areas of narrowing, saccular aneurysm, and its communication with adjacent cortical vein, which was seen draining into SSS anteriorly. The posterior tributary was seen coursing inferiorly to join the sylvian vein. Normal SSS

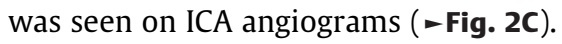

The patient had direct CVD and twice ruptured venous aneurysm (Cognard type IV, Borden type III). This vSSS DAVF was considered as aggressive pathology and planned treatment was TAE. Bilateral femoral arterial accesses were obtained. 5F Envoy guide catheter (Cordis, Santa Clara, CA) was positioned, through right femoral arterial access, in distal right ECA to guide Marathon microcatheter and Mirage microwire combination (Micro Therapeutics Inc, Irvine, California) into right MMA. The Marathon microcatheter tip was

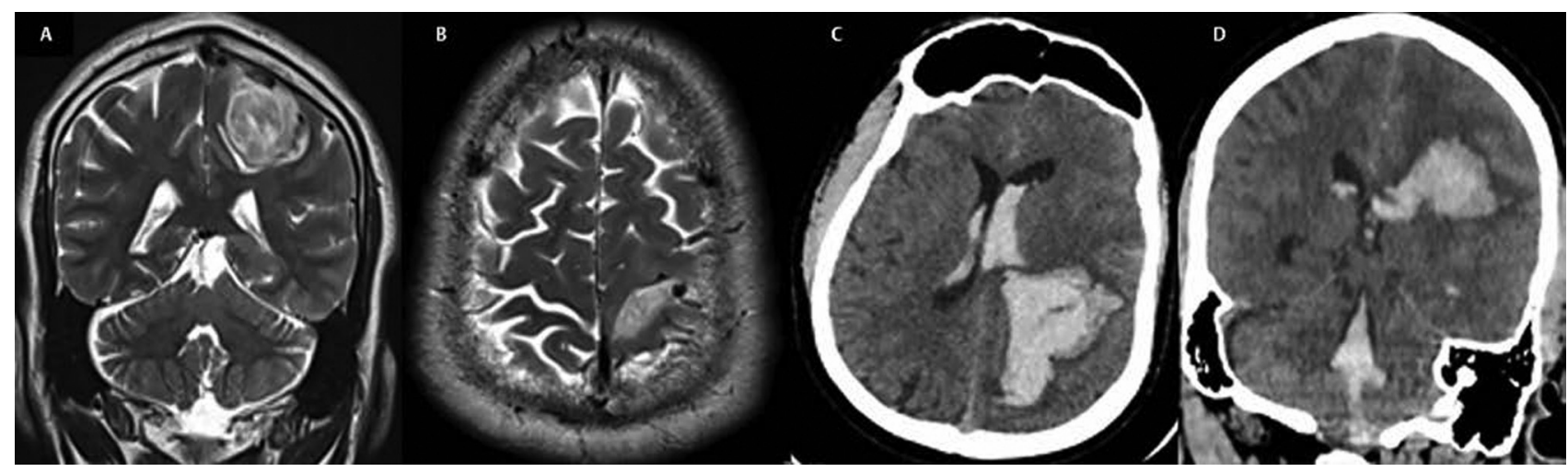

Fig. 1 MRI brain. (A) Coronal T2-weighted (T2W) image showing extent of the hematoma. (B) T2W axial image showing rounded flow void within the adjacent sulcal space close to hematoma. Repeat CT study (C) Axial (D) Coronal reformatted images revealing increased volume of hematoma, extending into ventricles. 


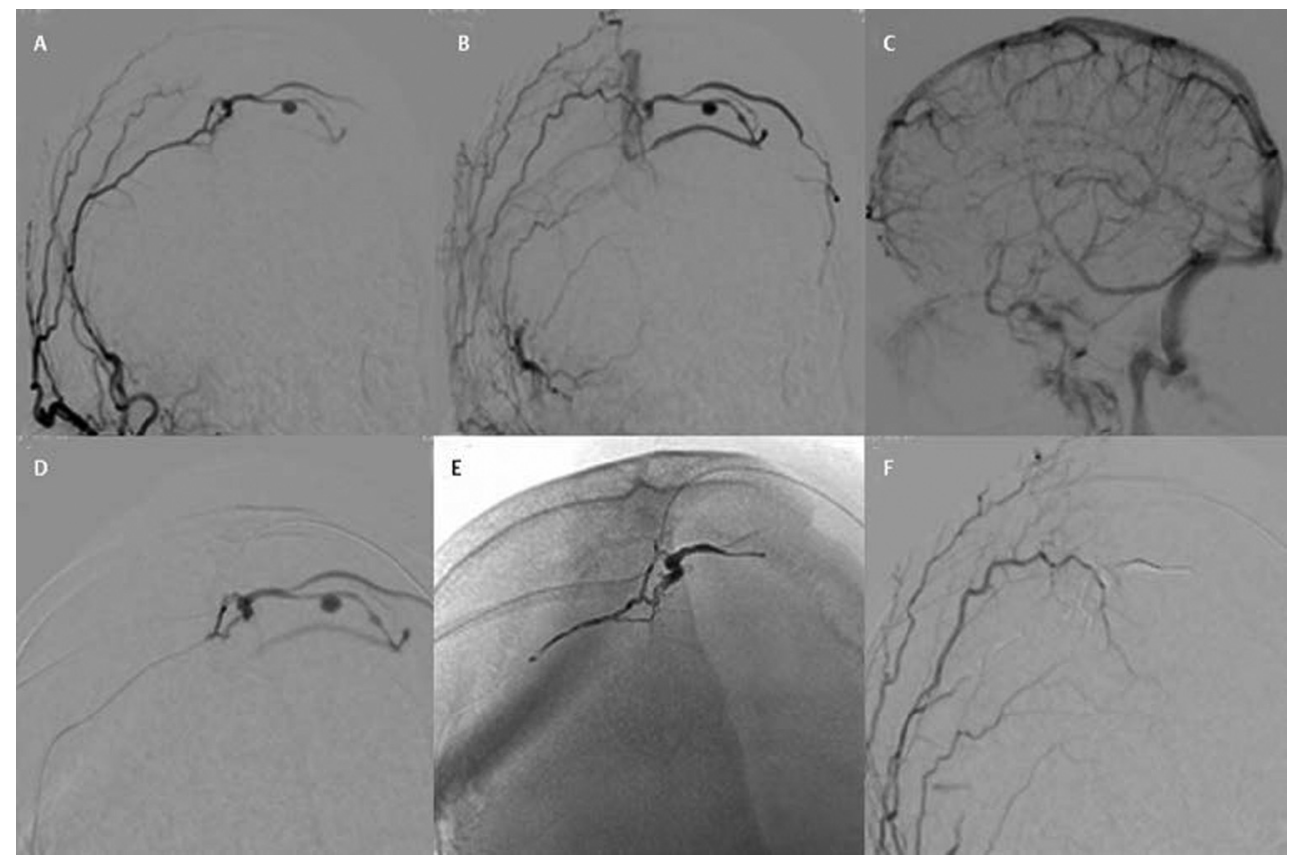

Fig. 2 Digital subtraction angiography (DSA) study right external carotid artery (ECA) angiogram right anterior oblique (RAO) projection (A, early arterial) and (B, delayed phase), showing fistula within the wall of patent superior sagittal sinus (SSS), fed by right middle meningeal artery (MMA) and draining into cortical vein. Patent SSS is seen surrounded by fistula (B). Venous aneurysm is seen on the anterior tributary, which joins SSS. (C) Internal carotid artery (ICA) angiogram, venous phase, showing normal SSS. (D) Microcatheter angiogram depicting feeders, fistula within the SSS wall, draining veins and venous aneurysm. (E) Onyx cast; note the Onyx did not reach venous aneurysm. (F) Right ECA oblique projection showing complete occlusion of fistula.

positioned just proximal to the DAVF ( - Fig. 2D). Onyx 18 (Micro Therapeutics Inc) was then slowly injected under continuous roadmap guidance. Effective Onyx cast was seen within the fistulous connections lying within SSS wall, with extension into feeder and draining veins (-Fig. 2E). Onyx was not injected into the venous aneurysm. Complete obliteration of the DAVF was confirmed on six vessel check angiographies, which were done through left femoral arterial access ( - Fig. 2F). Venous aneurysm was not opacified on any of these check angiograms. The patient continued to show neurological improvement and was discharged after 2 weeks. Follow-up six vessel digital subtraction angiography (DSA) after a few months (-Fig. 3A, B) revealed persistent obliteration of DAVF. The large venous aneurysm was not seen on follow-up DSA study/CT studies.

\section{Review of Literature}

\section{Methods}

A systematic search of English published articles was performed on PubMed. We used specific key words like "dural arteriovenous fistula," "superior sagittal sinus," "direct cortical venous drainage," "venous ectasia," "venous aneurysm," "Cognard type III fistula," "Cognard type IV

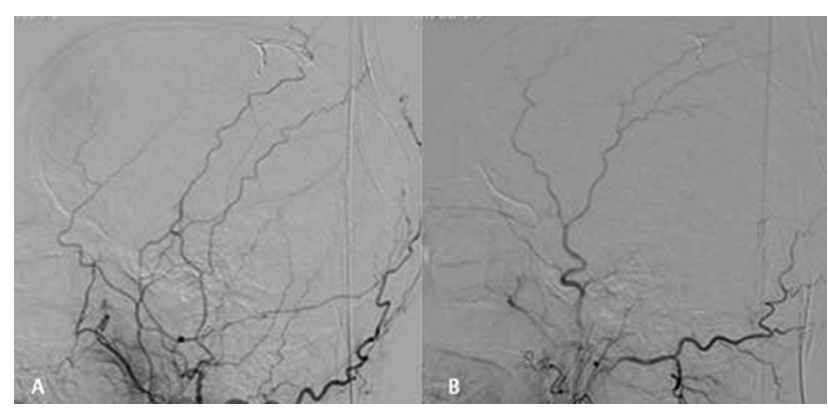

Fig. 3 Follow-up angiograms; lateral projections. (A) Right external carotid artery (ECA) (B) Left ECA showing complete obliteration of fistula. No recanalization seen.

fistula," "Borden type 3 fistula," "variant," in various combinations to identify the primary list of articles. Titles and abstracts were then analyzed to identify the potential articles. These potential articles were then reviewed, based on inclusion criteria.

The following inclusion criteria were used: A) type of dural fistula/direct cortical venous drainage was clearly mentioned; B) in Borden type 3 fistula, presence or absence of venous ectasia was mentioned; $C$ ) specific mention of SSS location was noted; D) details of the treatment offered were available; E) at 


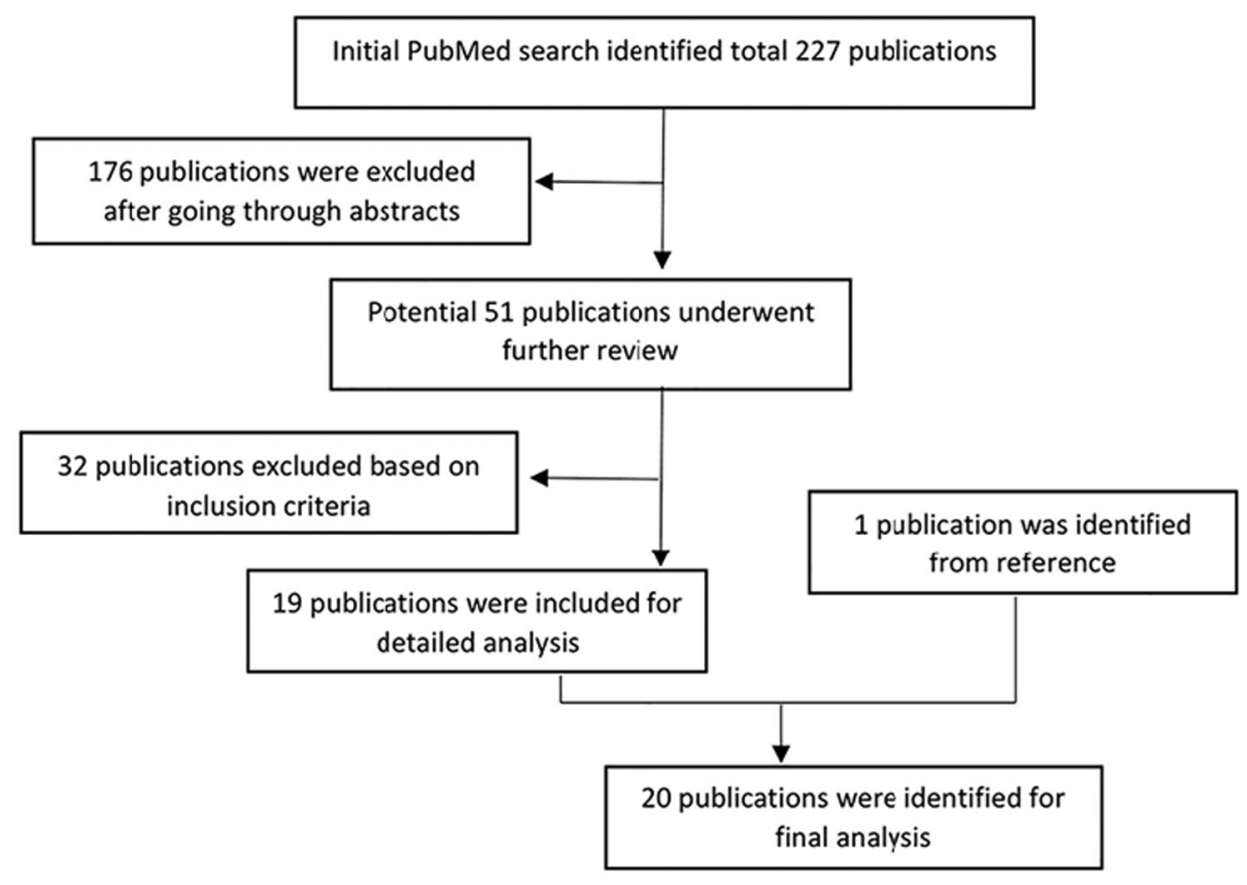

Fig. 4 Flow diagram of literature search.

least some details of clinical data and angiographic findings were available along with clinical follow-up. Cases from large series, which fulfilled these criteria, were also included. Records with incomplete information were excluded. Few cases reported as SSS involvement were found to have early opacification of sinus with contrast and then refluxing into cortical veins; we excluded such type 2 DAVF cases. Publications fulfilling inclusion criteria were systematically reviewed. Reference lists of all these selected articles were then manually checked for additional studies. The flow diagram of this search is shown in -Fig. 4.

\section{Results}

A total of 31 cases of vSSS DAVF were reported in 20 publications. ${ }^{2,5-8,10-24}$ We analyzed general demographic, clinical, radiological, angiographic, and treatment features of all these cases along with clinical outcome ( - Table 1 and - Table 2 ).

\section{Age, Sex}

Age of three patients and sex of one patient was not available. These SSS DAVFs are seen predominantly involving male patients in their sixth decade. They were not observed in the pediatric age group.

\section{Etiology, SSS Thrombus}

Previous details of trauma, sinus thrombosis, infection, and tumor were not available in six patients. Majority of
vSSS DAVF were idiopathic in nature (65.38\%). Seven patients, including the current patient, had a past history of head injury, making trauma a most likely etiological factor (26.92\%). One patient had meningioma, invading SSS with vDAVF seen just anterior to it. ${ }^{19}$ One patient was treated for bacterial meningitis a few months back. ${ }^{2}$ Surgery and underlying SSS thrombosis were not associated with vDAVF. SSS patency was not mentioned in six patients; most of the remaining patients (96.15\%) showed normal patent SSS on angiograms. Patient with meningioma had thrombosed SSS due to tumor invasion.

\section{Clinical Presentation and Imaging Findings}

These details were not available in one patient. Thirteen patients $(41.93 \%, 13 / 31)$ had ICH. In two patients, exact location of bleed was not mentioned. Eight patients $(25.80 \%, 8 / 31)$ had intraparenchymal hemorrhage (IPH), three patients $(9.67 \%, 3 / 31)$ had subarachnoid hemorrhage (SAH), two patients $(6.45 \%, 2 / 31)$ had intraventricular hemorrhage (IVH), and one patient $(3.22 \%, 1 / 31)$ had subdural hemorrhage (SDH). Two patients, including current case, had rebleed $(15.38 \%, 2 / 13)$. The current patient had IPH along with SAH initially and rebled soon after admission, with significant increase in IPH along with extensive IVH. A total of 24 patients $(77.41 \%, 24 / 31)$ had aggressive clinical presentation. In two patients, venous ischemia was demonstrated on single-photon emission computerized tomography (SPECT) study. ${ }^{2,8}$ 
Superior Sagittal Sinus Wall Fistula with Direct Cortical Venous Drainage Chavan et al. 123

\begin{tabular}{|c|c|c|c|c|c|c|c|c|c|c|c|c|c|c|}
\hline 厄. & 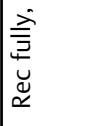 & 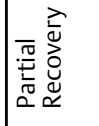 & 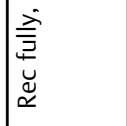 & 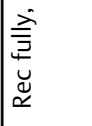 & 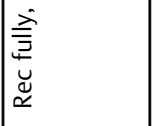 & 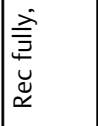 & 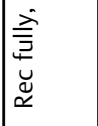 & 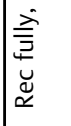 & 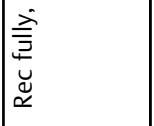 & 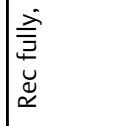 & $\Sigma$ & $\Sigma$ & $\Sigma$ & $\frac{\vec{z}}{\overline{\underline{u}}}$ \\
\hline $\begin{array}{l}\frac{9}{7} \\
\text { ì } \\
\overline{0} \\
\end{array}$ & $\begin{array}{l}\stackrel{n}{E} \\
\stackrel{+}{E} \\
m\end{array}$ & $\sum_{\sigma}^{\infty}$ & $\sum_{\nu}^{n}$ & $\begin{array}{l}8 \\
\dot{ \pm} \\
-\end{array}$ & 号 & $\sum_{\sigma}^{n}$ & $\sum_{m}^{n}$ & $\sum_{m}^{n}$ & \begin{tabular}{|l}
$\stackrel{n}{\underline{E}}$ \\
En \\
in
\end{tabular} & 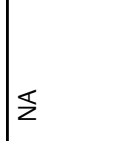 & $\Sigma$ & $\mathbb{z}$ & $\Sigma$ & $\stackrel{n}{\Sigma}$ \\
\hline हू & z & z & $\ddot{z}$ & $\ddot{z}$ & iz & z & $\ddot{z}$ & 2 & 2 & ì & z & iz & 2 & iz \\
\hline 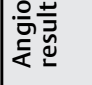 & ८ & 8 & 8 & 8 & 8 & ४ & 8 & 8 & 8 & 8 & ४ & 8 & 8 & 8 \\
\hline 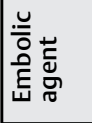 & 1 & $\stackrel{\$}{a}$ & 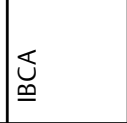 & 艾志 & 1 & $\begin{array}{l}\underset{\Phi}{\Phi} \\
\underline{\underline{a}}\end{array}$ & $\begin{array}{l}\infty \\
\mathbb{S}_{0} \\
=\end{array}$ & $\underset{Ð}{Ð}$ & 焉 & 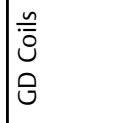 & 产 & 竘 & 状 & $\mid \begin{array}{l}\mathbb{s} \\
\text { 总 }\end{array}$ \\
\hline $5 \stackrel{\breve{u ̈ ~}}{5}$ & 1 & $\sum_{\Sigma}^{\mathbb{3}}$ & 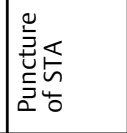 & 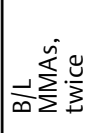 & 1 & 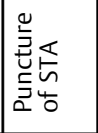 & $\sum_{\Sigma}^{\Sigma}$ & 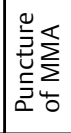 & 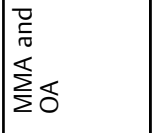 & $\vec{\gtrless}$ & $=\sum_{\infty}^{\infty}$ & $\sum_{\Sigma}^{\mathbb{L}}$ & $\sum_{\Sigma}^{\mathbb{L}}$ & $=\sum_{\infty}^{\infty} \sum^{\frac{n}{2}}$ \\
\hline 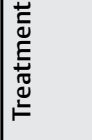 & $\begin{array}{l}\text { 离 } \\
\text { ज्ञ } \\
\text { ज }\end{array}$ & 5 & 5 & 点 & 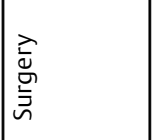 & 占 & 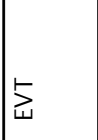 & 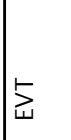 & 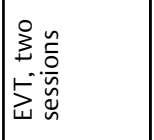 & 占 & 点 & 点 & 点 & 离 \\
\hline 츨 & $-\sum_{\infty}^{i=\frac{1}{\infty}}$ & 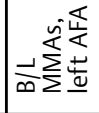 & 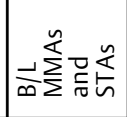 & 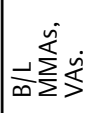 & 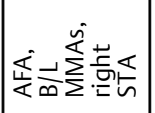 & $\Rightarrow \sum_{\infty}^{\infty} \sum_{i}^{\infty}$ & 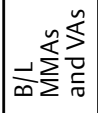 & $=\sum_{\infty}^{n}$ & - & 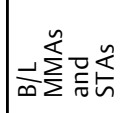 & $=\sum_{\infty}^{\frac{n}{x}}$ & 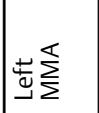 & 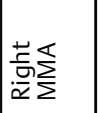 & 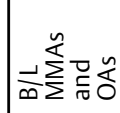 \\
\hline 离 & 2 & 2 & $\stackrel{\Perp}{\rightleftharpoons}$ & $\stackrel{\check{\nu}}{\rightleftharpoons}$ & $\stackrel{\ddot{\Perp}}{\succ}$ & 2 & z & 20 & $\stackrel{\check{\nu}}{\nu}$ & $\stackrel{\Perp}{\nu}$ & $\stackrel{u}{>}$ & z & $\stackrel{\check{\varpi}}{\rightleftharpoons}$ & 운 \\
\hline$\underline{\underline{I}}$ & 2 & 2 & 2 & 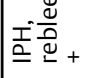 & 2 & 2 & 2 & 蒿 & $\underline{\underline{\underline{I}}}$ & 2 & $\underline{\underline{I}}$ & 䅃 & $\stackrel{I}{\underline{I}}$ & $\underline{\underline{I}}$ \\
\hline $\begin{array}{l}\text { केे } \\
\text { 응 }\end{array}$ & 趈 & 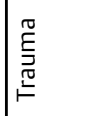 & 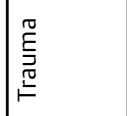 & 2 & 2 & 蛋 & 2 & 2 & 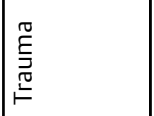 & 2 & 2 & 2 & 2 & z \\
\hline 芯莺 & a & $a$ & a & a & a & $a$ & a & o & a & a & a & a & a & a \\
\hline$\tilde{\tilde{n}}$ & $\tilde{\widehat{\Lambda}}$ & $\stackrel{\widetilde{\Upsilon}}{\Sigma}$ & $\stackrel{\widetilde{n}}{\stackrel{\tilde{N}}{\Sigma}}$ & $\stackrel{\widetilde{u}}{\Sigma}$ & $\begin{array}{l}\widetilde{\widetilde{n}} \\
\grave{\sim} .\end{array}$ & $\begin{array}{l}\tilde{u} \\
\vdots \\
\vdots\end{array}$ & 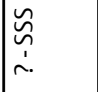 & $\begin{array}{l}\tilde{u} \\
\grave{n} \\
\end{array}$ & $\widetilde{\widetilde{\Lambda}}$ & 䒚 & 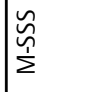 & $\mid \begin{array}{l}\tilde{u} \\
\grave{n} \\
\end{array}$ & $\begin{array}{l}\tilde{u} \\
\grave{\Lambda} \\
\end{array}$ & 苁 \\
\hline 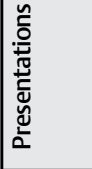 & 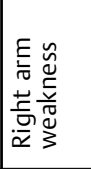 & 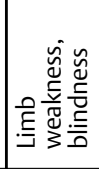 & 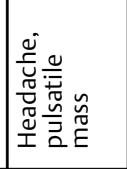 & 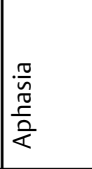 & 亗 & 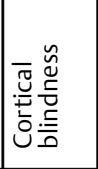 & 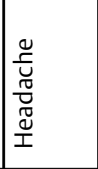 & I & 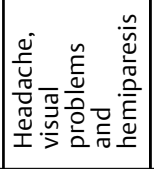 & 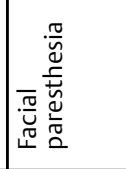 & 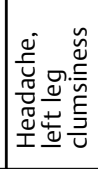 & 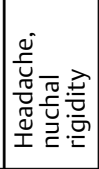 & 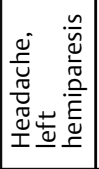 & 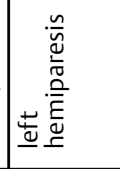 \\
\hline 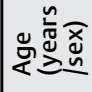 & $\sum_{0}^{0}$ & $\Sigma$ & $\Sigma$ & $\Sigma$ & $\frac{\mathrm{u}}{2}$ & $\sum_{\substack{\text { Hn } \\
\text { n }}}$ & 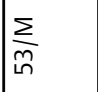 & $\frac{\stackrel{u}{\infty}}{\stackrel{\infty}{f}}$ & $\sum_{\substack{0\\
}}$ & 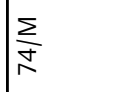 & $\sum_{\substack{\infty \\
m}}$ & $\frac{\sum}{\sigma}$ & $\sum_{\widehat{\sigma}}$ & $\sum_{i n}$ \\
\hline 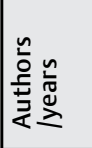 & 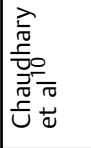 & 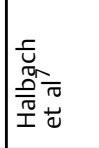 & 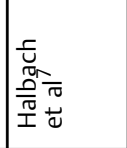 & 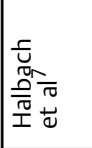 & 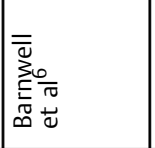 & 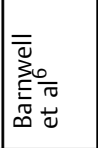 & 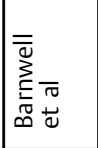 & 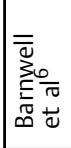 & 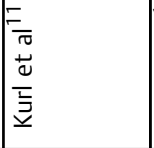 & 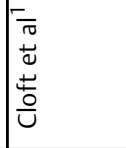 & 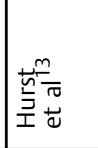 & 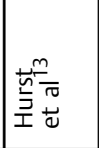 & $\begin{array}{l}\hbar^{m} \\
\tilde{y}^{\frac{m}{1}} \\
\frac{\pi}{d}\end{array}$ & $\mid$ \\
\hline $\begin{array}{l}\dot{\nu} \\
\tilde{\Xi} \dot{\Xi}\end{array}$ & - & $\sim$ & $m$ & $\nabla$ & in & 6 & $\curvearrowright$ & $\infty$ & $\sigma$ & $\circ$ & $=$ & $\simeq$ & $m$ & $\nexists$ \\
\hline
\end{tabular}




\begin{tabular}{|c|c|c|c|c|c|c|c|c|c|c|c|c|c|c|c|}
\hline 氖 & 彥 & 离 & 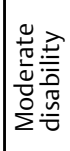 & 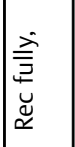 & $\Sigma$ & 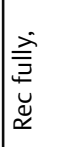 & 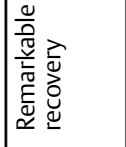 & 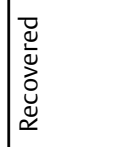 & 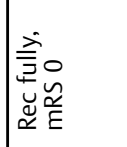 & 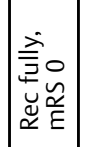 & 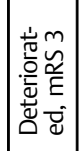 & 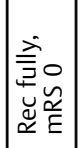 & 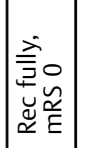 & 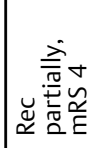 & $\ddot{\ddot{~}}$ \\
\hline 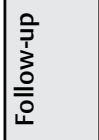 & $\begin{array}{l}\tilde{n} \\
\stackrel{\tilde{E}}{E} \\
6\end{array}$ & 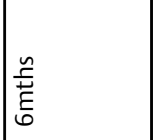 & 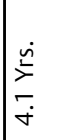 & $\Sigma$ & $\Sigma$ & $\Sigma$ & $\Sigma$ & z & $\begin{array}{l}\tilde{n} \\
\stackrel{\tilde{E}}{E} \\
\sim \\
N\end{array}$ & $\begin{array}{l}n \\
\underline{\underline{t}} \\
\underline{E} \\
\tilde{m}\end{array}$ & $\Sigma$ & $\Sigma$ & $\Sigma$ & 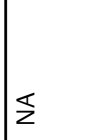 & $\Sigma$ \\
\hline 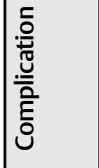 & 2 & 2 & 1 & 1 & 2 & z & $2 \frac{0}{2}$ & 2 & 2 & 2 & 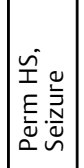 & 울 & 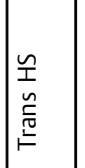 & 2 & 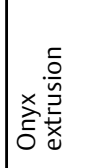 \\
\hline 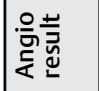 & ○ & ○ & 1 & 1 & ○ & ○ & ○ & $\frac{\rho}{\alpha}$ & ○ & ○ & ○ & ๑ & ○ & $\frac{\rho}{\alpha}$ & $\frac{\rho}{\alpha}$ \\
\hline 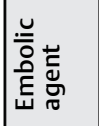 & $\sum_{\dot{m}}^{\infty} \sum_{\Sigma}^{\frac{1}{z}}$ & $\begin{array}{l}\frac{n}{0} \\
0 \\
0\end{array}$ & 1 & 1 & $\begin{array}{l}\check{n} \\
\overline{\bar{u}} \\
0\end{array}$ & $\begin{array}{l}\mathbb{x} \\
\text { 竞 }\end{array}$ & $\mid \begin{array}{l}\overleftarrow{s} \\
z\end{array}$ & 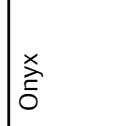 & 彥 & 㒷 & 1 & 1 & 1 & 1 & $\begin{array}{l}\infty \\
\frac{\infty}{x} \\
x_{\bar{E}}^{-}\end{array}$ \\
\hline 5 岀 & 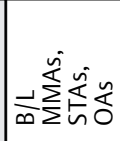 & 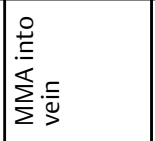 & 1 & 1 & 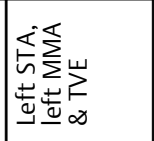 & 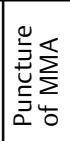 & 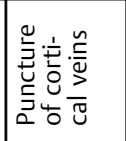 & 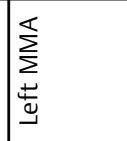 & 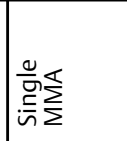 & 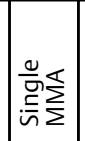 & 1 & 1 & 1 & 1 & 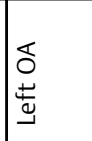 \\
\hline 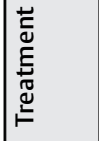 & 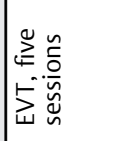 & 5 & 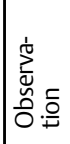 & 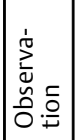 & 5 & 5 & 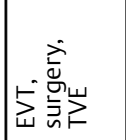 & 点 & 5 & 5 & $\begin{array}{l}\text { 离 } \\
\text { ज़ } \\
\bar{ज}\end{array}$ & 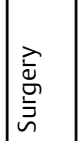 & 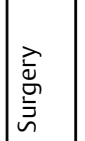 & 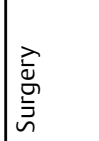 & 至离 \\
\hline $\begin{array}{l}\text { ⿳亠े⿵冂卄八 } \\
\text { ज्ञ }\end{array}$ & 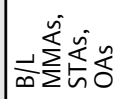 & 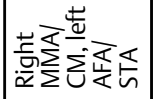 & $\Sigma$ & $\bar{z}$ & 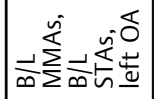 & $=\sum_{\infty}^{\frac{n}{\infty}}$ & 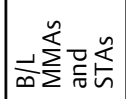 & $\Rightarrow \sum_{\infty}^{n} \sum_{i}^{n} \frac{n}{n}$ & 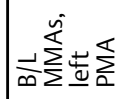 & 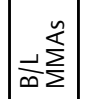 & zs & $\Sigma$ & z & z & $\frac{1}{\infty} \overbrace{}^{-\infty} \sum^{-\frac{5}{4}}$ \\
\hline $\begin{array}{l}\frac{\pi}{\tilde{n}} \\
\stackrel{\tilde{w}}{W}\end{array}$ & 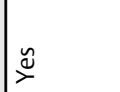 & $\stackrel{\nu}{\nu}$ & 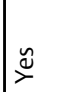 & z & $\Longleftarrow$ & $\stackrel{\check{\Perp}}{\rightleftharpoons}$ & $\stackrel{\check{\nu}}{\check{\nu}}$ & 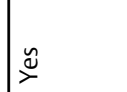 & $\stackrel{\check{\nu}}{\check{\nu}}$ & $\stackrel{\check{a}}{\rightleftharpoons}$ & z & q & z & z & z \\
\hline$\underline{I}$ & ì & z & z & z & z & zo & z & z & z & $\underline{\underline{I}}$ & ż & zo & 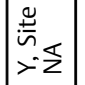 & 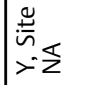 & z \\
\hline 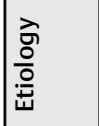 & zo & 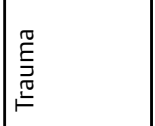 & z & $\Sigma$ & z & 2 & 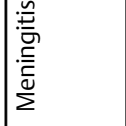 & 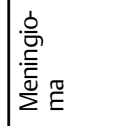 & z & 2 & $\S$ & z & $\Sigma$ & 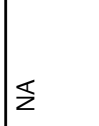 & z \\
\hline$\tilde{\varkappa}$ & a & o & z & a & a & a & a & 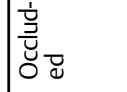 & a & a & $\S$ & $\bar{z}$ & $\Sigma$ & $\Sigma$ & $\Sigma$ \\
\hline$\tilde{\varkappa}$ & 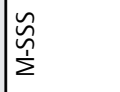 & 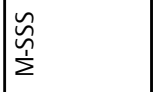 & $\begin{array}{l}\tilde{u} \\
\vdots \\
r\end{array}$ & $\begin{array}{l}\tilde{u} \\
\vdots \\
.\end{array}$ & $\dot{a}_{\dot{1}}^{\prime} \tilde{u}$ & $\mid \begin{array}{l}\tilde{\hat{\alpha}} \\
\sum\end{array}$ & 苍 & $\sum_{\tilde{c}}^{\tilde{c}}$ & $\widetilde{\widetilde{c}}$ & $\underline{\varkappa}$ & $\stackrel{\widetilde{\varkappa}}{\tilde{c}}$ & 气ૂ. & 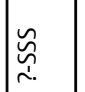 & 峞. & 荇 \\
\hline 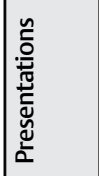 & 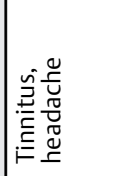 & 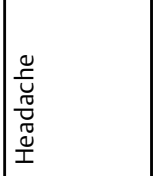 & z & 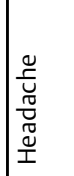 & 㺃 & 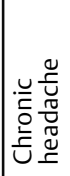 & 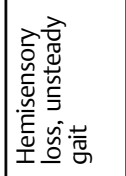 & 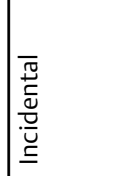 & 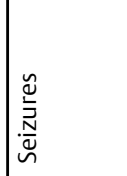 & 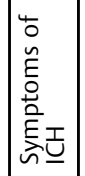 & 高 & 亗 & 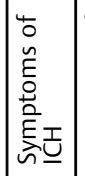 & 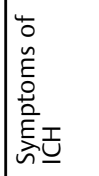 & 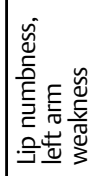 \\
\hline 点 & 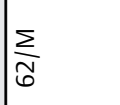 & $\frac{u}{\infty}$ & 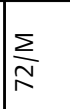 & $\sum_{\substack{6 \\
q}}$ & $\sum_{\text {in }}$ & $\sum_{\widehat{6}}$ & $\mid \frac{\Sigma}{g}$ & $\mid \begin{array}{l}z \\
0\end{array}$ & 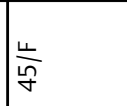 & 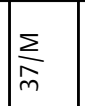 & $\frac{\sum}{6}$ & 翙 & $\frac{\mathrm{U}}{\mathbf{N}}$ & 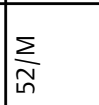 & $\mid \begin{array}{l}\Sigma \\
⿱ \\
0 \\
0\end{array}$ \\
\hline 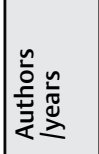 & 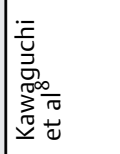 & $\mid \frac{n}{\sqrt[n]{\frac{n}{\sigma}}}$ & 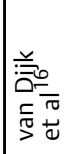 & 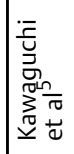 & 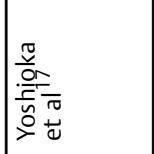 & $\begin{array}{l}\frac{\infty}{\pi} \\
\frac{0}{\pi} \\
+\frac{0}{0} \\
\frac{\sigma}{0} \\
\underline{0}\end{array}$ & 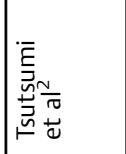 & 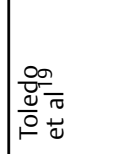 & 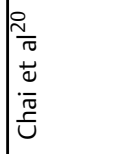 & 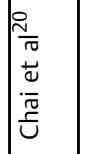 & $\mid$ & $\mid$ & 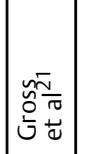 & 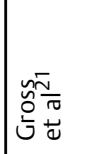 & 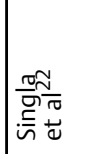 \\
\hline 岕̊ & $\stackrel{\text { ㄴ }}{ }$ & 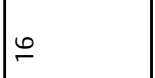 & 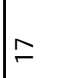 & $\stackrel{\infty}{-}$ & 9 & i & $\bar{N}$ & N & $\ddot{\sim}$ & $\stackrel{\text { N }}{ }$ & $\stackrel{\Perp}{\sim}$ & $\stackrel{2}{N}$ & 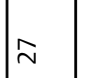 & $\stackrel{\infty}{\sim}$ & શิ \\
\hline
\end{tabular}




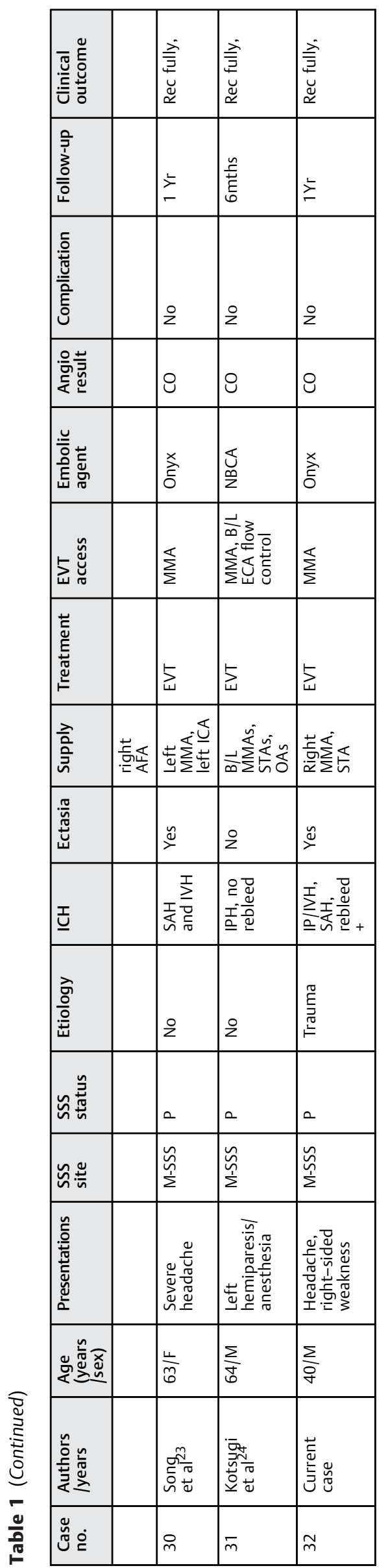

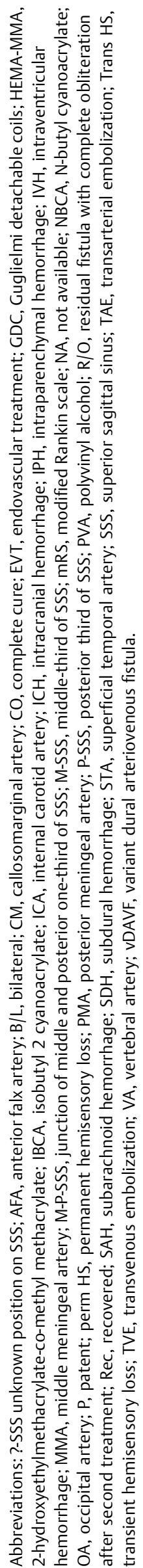

\section{Location}

Exact location of fistula on SSS was not mentioned in 12 patients. Middle one-third of SSS was the most common location (75\%, 15/20). None showed involvement of anterior SSS.

\section{Arterial Feeders}

Details of the arterial feeders were not available in 6 patients (18.75\%, 6/32). MMA was the most common artery supplying these vDAVFs, seen in all 26 patients (100\%, 26/26), followed by STA and occipital arteries

\section{Venous Ectasias}

A total of 19 patients $(59.37 \%, 19 / 32)$ showed changes of venous ectasias; such changes were absent in 13 patients $(40.62 \%, 13 / 32)$. Song et al reported three aneurysms on the draining venous pathway, claiming to be a maximum in the literature. $^{23}$ The current patient had a saccular venous aneurysm, which bled twice in a short span of time.

\section{Treatment Modalities}

Two patients $(6.25 \%, 2 / 32)$ were observed without any treatment. Endovascular treatment (EVT) completely cured fistula in 20 patients. In two patients, who underwent TAE, STA was punctured percutaneously and used as an access. 6,7 In two patients, MMA was surgically exposed, punctured, and used as access for TAE. ${ }^{6,18}$ In one patient, draining cortical veins were punctured transcranially, after craniotomy, to inject N-butyl cyanoacrylate (NBCA). ${ }^{2}$ Only TVE was done in one patient. ${ }^{12}$ One patient had TAE to reduce the blood flow, followed by TVE to occlude the venous pouch. ${ }^{17}$ MMA was the most common access used for EVT (79.16\%, 19/24).

\section{Embolic Agents}

In 10 patients $(41.66 \%, 10 / 24)$, either NBCA or isobutyl cyanoacrylate (IBCA) was used. Combination of polyvinyl alcohol (PVA) particles and NBCA or IBCA was used in three patients $(12.5 \%, 3 / 24)$. Only PVA particles were used in one patient. Selectively detachable coils were used in three patients. 2-hydroxyethyl methacrylate-co-methyl methacrylate (HEMA-MMA) was used as embolic agent in one patient, while Onyx liquid embolic agent was used in six patients. Embolization was performed through single feeder artery when Onyx was used as embolic agent, while catheterization of multiple feeders and multiple sessions were required for other embolic agents (PVA, NBCA, IBCA, HEMAMMA). Balloon guide catheters have been used in bilateral external carotid arteries to control blood flow during embolization with NBCA. ${ }^{24}$

\section{Complications}

Two patients developed neurological complications after surgical treatment. One patient developed transient hemisensory deficit, while another patient had permanent hemisensory deficits. No patient, who underwent EVT options, developed immediate neurological complications; however, one patient had delayed Onyx extrusion into the scalp subcutaneous region. ${ }^{22}$ 
126 Superior Sagittal Sinus Wall Fistula with Direct Cortical Venous Drainage Chavan et al.

Table 2 Clinical, radiological, angiographic and treatment features of SSS vDAVFs

\begin{tabular}{|c|c|c|}
\hline \multirow[t]{3}{*}{ Demographics } & Sex, male & $77.41 \%, 24 / 31$ \\
\hline & Age, range (years) $(\%, n / N)$ & 19 to 74 \\
\hline & Age, average (years) & 54 \\
\hline \multirow[t]{2}{*}{ Etiology } & History of head injury $(\%, n / N)$ & $26.92 \%, 7 / 26$ \\
\hline & Idiopathic $(\%, n / N)$ & $65.38 \%, 17 / 26$ \\
\hline \multirow[t]{2}{*}{ SSS status } & Patent & $96.15 \%, 25 / 26$ \\
\hline & Not mentioned & $18.75 \%, 6 / 32$ \\
\hline \multirow[t]{6}{*}{ Clinical presentation } & $\mathrm{ICH}$ & $41.93 \%, 13 / 31$ \\
\hline & Headache & $38.70 \%, 12 / 31$ \\
\hline & Hemiparesis/limb weakness & $35.48 \%, 11 / 31$ \\
\hline & Seizures & $12.90 \%, 4 / 31$ \\
\hline & Visual symptoms & $9.67 \%, 3 / 31$ \\
\hline & Gait disturbance, tinnitus, dementia, aphasia & $3.22 \%, 1 / 31$ Each \\
\hline \multirow[t]{4}{*}{ Location on SSS } & Not mentioned & $37.50 \%, 12 / 32$ \\
\hline & Middle one-third & $75 \%, 15 / 20$ \\
\hline & Posterior one-third & $20 \%, 4 / 20$ \\
\hline & Middle and posterior junction & $5 \%, 1 / 20$ \\
\hline \multirow[t]{9}{*}{ Arterial feeders } & B/L MMAs & $80.76 \%, 21 / 26$ \\
\hline & B/L STAs & $30.76 \%, 8 / 26$ \\
\hline & $\mathrm{B} / \mathrm{L} \mathrm{OAs}$ & $19.23 \%, 5 / 26$ \\
\hline & $\mathrm{B} / \mathrm{L}$ vertebral arteries & $7.69 \%, 2 / 26$ \\
\hline & Unilateral MMA & $19.23 \%, 5 / 26$ \\
\hline & Unilateral STA & $11.53 \%, 3 / 26$ \\
\hline & Unilateral vertebral artery & $3.84 \%, 1 / 26$ \\
\hline & Unilateral occipital artery & $7.69 \%, 2 / 26$ \\
\hline & AFA & $15.38 \%, 4 / 26$ \\
\hline \multirow[t]{2}{*}{ Venous ectasia } & Ectasia present & $59.37 \%, 19 / 32$ \\
\hline & Ectasia absent & $40.62 \%, 13 / 32$ \\
\hline \multirow[t]{5}{*}{ Treatment modalities } & Only EVT & $62.50 \%, 20 / 32$ \\
\hline & Surgery without EVT & $18.75 \%, 6 / 32$ \\
\hline & Surgery with prior EVT & $12.5 \%, 4 / 32$ \\
\hline & No treatment & $6.25 \%, 2 / 32$ \\
\hline & MMA access & $79.16 \%, 19 / 24$ \\
\hline \multirow[t]{5}{*}{ Embolic agents used } & PVA, HEMA-MMA & $4.16 \%, 1 / 24$ \\
\hline & Either NBCA or IBCA & $41.66 \%, 10 / 24$ \\
\hline & PVA with NBCA or IBCA & $12.5 \%, 3 / 24$ \\
\hline & GDC & $12.5 \%, 3 / 24$ \\
\hline & Onyx & $25 \%, 6 / 24$ \\
\hline \multirow[t]{2}{*}{ Outcome } & Angiographic cure & $100 \%, 30 / 30$ \\
\hline & Clinical recovery & $92.30 \%, 24 / 26$ \\
\hline
\end{tabular}

Abbreviations: AFA, anterior falx artery; B/L, bilateral; EVT, endovascular treatment; GDC, Guglielmi detachable coils; HEMA-MMA, 2-hydroxyethylmethacrylate-co-methyl methacrylate; IBCA, isobutyl 2 cyanoacrylate; ICH, intracranial hemorrhage; MMA, middle meningeal artery; NBCA, Nbutyl cyanoacrylate; OA, occipital artery; PVA, polyvinyl alcohol; STA, superficial temporal artery; SSS, superior sagittal sinus; vDAVF, variant dural arteriovenous fistula. 


\section{Angiographic and Clinical Outcome}

All patients with vDAVFs, who underwent any kind of treatment, experienced complete angiographic cure $(100 \%$, 30/30). No patient had residual DAVF. Clinical outcome of six patients was not available. One patient, who developed permanent hemisensory deficit, showed further deterioration at 3-year visit. One patient, who was not offered any curative treatment, showed moderate disability. The rest of the patients $(92.30 \%, 24 / 26)$ showed recovery on follow-up clinical visits.

\section{Discussion}

DAVFs are acquired with AV fistulous connections lying within venous sinus walls. The main arterial supply comes from meningeal branches of the ECA, with occasional supply from the ICA and vertebral arteries. ${ }^{13}$ Ingrowth of vessels adjacent to sinus thrombus leads to AV fistula formation. If the adjacent sinus is patent, then outflow is maintained through the sinus. ${ }^{6}$ Djindjian and Merland first pointed out association of sinus thrombosis preceding DAVF development. ${ }^{25}$ Studies reported retrograde transmission of venous hypertension, dilatation of vessels, and increased angiogenesis, leading to fistula formation. ${ }^{19,26-28}$ DAVFs most commonly involve transverse-sigmoid sinuses and cavernous sinuses. ${ }^{7}$ The location of a DAVF and its venous drainage pattern determines clinical presentation, bleeding risk, and prognosis. SSS DAVFs are relatively uncommon and represent approximately 8 to $11 \%$ of all DAVFs. ${ }^{4,7}$

Few DAVFs located within the wall of the SSS drain directly into cortical/leptomeningeal veins. These are Borden type 3, Cognard type III or type IV fistulas. These DAVFs are considered aggressive/high-grade fistulas. Direct transmission of high arterial shunt pressure to cortical veins causes various anatomical changes like bending and winding of veins and formation of venous varices/aneurysms. ${ }^{5}$ These lead to aggressive neurological presentations like ICH, venous infarct, and brain edema. ${ }^{17}$ Draining vein is labeled as ectatic when the venous diameter is larger than $5 \mathrm{~mm}$ or three times wider than the draining vein diameter. ${ }^{4}$ In the current case, the saccular outpouching measured $6 \times$ $4.8 \mathrm{~mm}$. Pathogenesis of development of such vDAVF is unknown; two theories have been postulated. ${ }^{14} \mathrm{~A}$ ) Complete recanalization of initially thrombosed adjacent sinus by ingrowing arterioles during the thrombus organizing process. Entrance point of cortical vein is still occluded by thrombus, leading to development of variant fistula. B) Thrombophlebitic process only involving entry point of cortical vein with normal sinus, which then recanalizes via arterioles from dural wall. Hamada et al histologically studied arterialized veins and venous aneurysms in DAVF patients. ${ }^{29}$ Venous aneurysms in cases with venous hypertension revealed uniform wall thickness, medial and intimal thickening, with loss of internal elastic lamina (IEL). In contrast, venous aneurysms in patients with hemorrhage showed extremely irregular wall thickness, no clear demarcation between media and intima, scant muscle tissue, and complete disappearance of IEL. More pronounced changes in patients with bleed were considered due to complex interplay of anatomical, hemodynamic, and degenerative factors.

These DAVFs show a male predominance. ${ }^{23}$ These can have IPH, SAH, SDH, IVH, venous infarct, or brain edema. Parenchymal hemorrhages are often surrounded by excessive vasogenic edema, due to venous hypertension, similar to metastatic brain deposits. ${ }^{13}$ Two studies have demonstrated low perfusion in the adjacent parenchyma due to venous hypertension, considered to indicate increased risk of bleed and neurological deficits. ${ }^{2,8}$ Underlying SSS may be thrombosed, may show signs of old thrombosis, or may be absolutely normal. In the current case, SSS was normal. On imaging, meningioma invariably shows prominent vascular flow voids; however, a possibility of associated DAVF also needs to be considered. ${ }^{19}$ Six vessel detailed angiography should be performed in all suspected patients with vDAVF.

The rate of bleeding with DAVFs is estimated at approximately $1.8 \%$ per year, with a relatively higher rate of bleed noted in aggressive DAVFs. ${ }^{3,4,30}$ Presence of leptomeningeal drainage and dysplastic venous dilatation is highly suggestive of increased risk of $\mathrm{ICH}^{30-32}$ Factors such as direct cortical venous drainage, venous ectasia or aneurysm, outflow restrictions, and long circuitous venous pathway define the increased hemorrhagic risk in vDAVFs. ${ }^{6,7}$ Bulters et al noted annual rates of hemorrhage of $3.5 \%, 13 \%$ and $27 \%$ in groups of patients of DAVFs with absent CVR, with CVR and with venous ectasia, respectively. ${ }^{33}$ Thus, the presence of venous ectasia increases risk of hemorrhage by seven-fold. Compared with Borden 2 lesions, Borden 3 lesions had double the rate of hemorrhage. Bleeding risk associated with type 3 SSS DAVF, due to rupture of draining veins, is up to $40 \%$, which is significantly higher than the DAVFs located at other sinuses. ${ }^{4,7,11}$

Rate of rebleeding was initially thought to be rare, even in patients presenting with $\mathrm{ICH}^{34-36}$ However, Borden et al showed rebleed in three of seven patients, who initially had ICH. They concluded that DAVFs presenting with ICH must be treated quickly. ${ }^{3}$ Duffau et al published a series of 20 patients with DAVFs with cortical venous drainage and $\mathrm{ICH}^{37}$ Out of these 20 patients, seven patients had rebleed within 2 weeks (35\%). Maximum rebleed occurred within the first week of initial bleed. Four out of these seven patients already had clinical features suggestive of hemorrhage prior to admission. All patients with rebleed were found comatose. The authors concluded that due to high rate of rebleed, grave clinical consequences, all patients of DAVFs with CVD should be treated as soon as possible. Van Dijk et al reported death of nine patients (45\%) in a series of 20 patients of DAVF with CVD who were either not treated or partially treated. ${ }^{16}$ Seven patients (35\%) had hemorrhage during follow-up period, while six patients (30\%) had nonhemorrhagic neurological 
deficits (NHND). Four patients developed progressive dementia due to venous hypertension, leading to death in three patients and leaving one patient in a moribund state. In this series, the annual mortality rate was $10.4 \%$ and combined annual hemorrhage and NHND rate was $15 \%$, mandating urgent cure. Bulters et al showed hemorrhage rate of $13 \%$ in untreated fistulae and $4.7 \%$ in partially treated patients. ${ }^{33}$ They noted that there was no hemorrhage in the group of partially treated patients in the first 2 years. This transient reduction in bleeding risk may be due to reduction in venous pressure, before new feeders were recruited, causing restoration of pathological venous pressure. However, the authors did not recommend waiting to treat these patients. Our patient rebled within a few hours.

Treatment of the DAVF is justified by comparing its natural course and risk involved in its proposed treatment. Having documented high rates of bleed and rebleed, these type 3 DAVFs demand urgent treatments. Compression therapy is not advised in these aggressive vDAVFs. ${ }^{6}$ Endovascular embolization and open surgery are available options to treat these DAVFs. Embolic agents causing permanent closure of fistula are preferred. ${ }^{13}$ Liquid embolic agents like IBCA, NBCA, HEMA-MMA, and Onyx lead to better outcomes versus PVA. In TAE approach, microcatheter is navigated close to the fistula, to increase chances of embolic agent obliterating actual fistulous connections, rather than proximal feeders. Transarterial NBCA embolization required multiple sessions with multiple-feeder access to achieve complete cure. ${ }^{6,7}$ Directly accessing the feeder arteries, by percutaneous puncture, has also been used. Two groups reported a case each of direct percutaneous puncture of STAs ${ }^{6,7}$ Direct access of MMA have also been used after craniotomy. ${ }^{6,18}$ All cases had complete resolution of DAVFs. Kawaguchi et al used HEMA-MMA in VDAVF with complete cure; however, five sessions were required. ${ }^{8}$

Although TVE is widely used to treat other types of DAVF, it is generally avoided while treating type 3 SSS Dural AVFs. Many treating physicians feel that transvenous access to traverse dilated tortuous cortical veins is very risky. It carries a risk of worsening patient's condition by obliterating venous outflow without occluding the fistulous nidus. ${ }^{12}$ There is also potential risk of reflux of embolic agents into the SSS, leading to disaster. Hence, TVE should be attempted with extreme care, only after ruling out other options. Cloft et al reported the first case of occlusion of parasagittal venous pouch in such DAVF via venous access. ${ }^{12}$ Yoshioka et al reported a case of complete cure in similar DAVF, wherein TAE was used to reduce blood flow, followed by transvenous occlusion of draining varicose vein with Guglielmi detachable coils (GDC). ${ }^{17}$ This is analogous to the surgical technique of simply ligating draining cortical veins at their entry into the subarachnoid space. ${ }^{34,38,39}$ Surgical resection is difficult in middle and posterior thirds of SSS, which drain majority of cerebral hemispheric venous outflow. ${ }^{11,21}$ Radiosurgery treatment option has been used to treat various DAVFs, however it is not advised in aggressive DAVF, due to signifi- cant delay of 1 to 3 years in complete cure. ${ }^{40,41}$ Spontaneous disappearance of venous aneurysms has been mentioned previously. ${ }^{23}$ Venous aneurysm in the current case was not seen on follow-up studies.

\section{Limitations}

Borden Type 3, Cognard type III/IV DAVFs on SSS are relatively rare intracranial vascular pathologies. Most of these cases, which we could collect, are published as case reports. Statistical analysis is inappropriate due to small sample size in our review. Due to our selection criteria, we could have missed cases without adequate details, especially cases included in large series. We have searched only English language publications, which might have resulted in missing out cases published in other languages. Because of all these limitations, our literature review might not project actual incidence of this rare intracranial vascular pathology.

\section{Conclusion}

Type III/IV DAVFs on the SSS are uncommon forms of DAVFs. They predominantly involve male patients. The middle-third of the SSS is the most common location, followed by the posterior one-third. Most of these fistulas are idiopathic and are not preceded by SSS thrombosis; trauma is a common etiological factor in the rest of the cases. The most common arterial supply comes from MMAs, usually bilateral, followed by STAs and occipital arteries (OAs). These fistulas have aggressive presentation because of direct cortical venous drainage, formation of venous aneurysms, venous outflow restrictions, and long venous course. Most of these patients present with ICH and NHND. Incidence of rebleed is also very high, up to $40 \%$, and warrants urgent treatment of these fistulas. Endovascular embolization, either transarterial or transvenous, open surgical treatment and their combination are available treatment strategies. Complete cure of the fistula is the aim, in order to prevent recurrence of bleed. Most of the patients have good outcomes, provided proper treatment is planned with complete angiographic cure and followed-up regularly.

\section{Financial Support}

We have not received any financial grant for this study.

Patient Consent and Hospital Ethics Committee Approval We received written informed consent from the patient. We also received approval from Hospital Ethics committee for collection, analysis, and publication of data of this patient.

\section{PRISMA Checklist}

Available as supplementary file.

Conflict of Interest

The authors declare that there is no conflict of interest. 
Acknowledgments

We would like to thank Ms. Vaishali Kulkarni and Mr. Ratnakar Kulkarni for all their contribution.

\section{References}

1 Newton TH, Cronqvist S. Involvement of dural arteries in intracranial arteriovenous malformations. Radiology 1969;93(05): 1071-1078

2 Tsutsumi S, Yasumoto Y, Ito M, Oishi H, Arai H, Yoritaka A. Atypical dural arteriovenous fistula associated with meningitis. Neurol Med Chir (Tokyo) 2008;48(02):68-71

3 Borden JA, Wu JK, Shucart WA. A proposed classification for spinal and cranial dural arteriovenous fistulous malformations and implications for treatment. J Neurosurg 1995;82(02):166-179

4 Cognard C, Gobin YP, Pierot Let al.Cerebral dural arteriovenous fistulas: clinical and angiographic correlation with a revised classification of venous drainage. Radiology 1995;194(03): 671-680

5 Kawaguchi T, Nakatani M, Kawano T. Study of dural arteriovenous fistula drains into leptomeningeal vein without sinus interposition. Interv Neuroradiol 2004;10(Suppl 1):127-134

6 Barnwell SL, Halbach VV, Dowd CF, Higashida RT, Hieshima GB, Wilson CB. A variant of arteriovenous fistulas within the wall of dural sinuses. Results of combined surgical and endovascular therapy. J Neurosurg 1991;74(02):199-204

7 Halbach VV, Higashida RT, Hieshima GB, Rosenblum M, Cahan L. Treatment of dural arteriovenous malformations involving the superior sagittal sinus. AJNR Am J Neuroradiol 1988;9(02): 337-343

8 Kawaguchi T, Kawano T, Kaneko Yet al.Transarterial embolization with HEMA-MMA of variant convexity-superior sagittal sinus dural arteriovenous fistula-case report. Neurol Med Chir (Tokyo) 2000;40(07):366-368

9 Hou K, Ji T, Guo Y, Xu B, Xu K, Yu J. Current status of endovascular treatment for dural arteriovenous fistulas in the superior sagittal sinus region: a systematic review of the literature. World Neurosurg 2019;122:133-143

10 Chaudhary MY, Sachdev VP, Cho SH. Weitzner I Jr, Puljic S, Huang YP. Dural arteriovenous malformation of the major venous sinuses: an acquired lesion. AJNR Am J Neuroradiol 1982;3(01): 13-19

11 Kurl S, Saari T, Vanninen R, Hernesniemi J. Dural arteriovenous fistulas of superior sagittal sinus: case report and review of literature. Surg Neurol 1996;45(03):250-255

12 Cloft HJ, Kallmes DF, Jensen JE, Dion JE. Percutaneous transvenous coil embolization of a type 4 sagittal sinus dural arteriovenous fistula: case report. Neurosurgery 1997;41(05):1191-1193

13 Hurst RW, Marcotte P, Raps EC, Flamm ES. Dural arteriovenous fistulas involving the superior sagittal sinus: acute presentation with intracranial hemorrhage. Surg Neurol 1998;49(01):42-46

14 Bavinzski G, Schoeggl A, Gruber A, Killer M. Variant arteriovenous fistula of the superior sagittal sinus-case report. Neurol Med Chir (Tokyo) 1999;39(05):362-366

15 Fukai J, Terada T, Kuwata Tet al.Transarterial intravenous coil embolization of dural arteriovenous fistula involving the superior sagittal sinus. Surg Neurol 2001;55(06):353-358

16 van Dijk JM, terBrugge KG, Willinsky RA, Wallace MC. Clinical course of cranial dural arteriovenous fistulas with long-term persistent cortical venous reflux. Stroke 2002;33(05):1233-1236

17 Yoshioka T, Kitagawa N, Yokoyama H, Nagata I. Selective transvenous coil embolization of dural arteriovenous fistula. A report of three cases. Interv Neuroradiol 2007;13(Suppl 1):123-130
18 Koh JS, Ryu CW, Bang JSet al.Transcranial approach for arterial embolization of dural arteriovenous fistula within the wall of the superior sagittal sinus: a case report. Neurointervention 2007; 2:117-121

19 Toledo MM, Wilson TJ, Dashti S, McDougall CG, Spetzler RF. Dural arteriovenous fistula associated with superior sagittal sinus occlusion secondary to invasion by a parafalcine meningioma: case report. Neurosurgery 2010;67(01):205-207

20 Chai EQ, Wang J. Transarterial Onyx embolization of sagittal sinus dural arteriovenous fistulae. Neurol India 2011;59(02):262-265

21 Gross BA, Du R. Surgical treatment of high grade dural arteriovenous fistulae. J Clin Neurosci 2013;20(11):1527-1532

22 Singla A, Fargen KM, Hoh B. Onyx extrusion through the scalp after embolization of dural arteriovenous fistula. J Neurointerv Surg 2016;8(09):e38

23 Song W, Sun H, Liu J, Liu L, Liu J. Spontaneous resolution of venous aneurysms after transarterial embolization of a variant superior sagittal sinus dural arteriovenous fistula: Case report and literature review. Neurologist 2017;22(05):186-195

24 Kotsugi M, Nakagawa I, Takamura Y, Wada T, Kichikawa K, Nakase H. Transarterial embolization of dural arteriovenous fistula in superior sagittal sinus under bilateral extrenal carotid artery flow control: Technical note. World Neurosurg 2019; 130:227-230

25 Djindjian R, Merland JJ. Superselective Arteriography of the External Carotid Artery. New York:. Springer 1978:405-412

26 Houser OW, Campbell JK, Campbell RJ. Sundt TM Jr. Arteriovenous malformation affecting the transverse dural venous sinus-an acquired lesion. Mayo Clin Proc 1979;54(10):651-661

27 Terada T, Higashida RT, Halbach VVet al.Development of acquired arteriovenous fistulas in rats due to venous hypertension. J Neurosurg 1994;80(05):884-889

28 Shin Y, Uranishi R, Nakase H, Sakaki T. Vascular endothelial growth factor expression in the rat dural arteriovenous fistula model. No To Shinkei 2003;55(11):946-952

29 Hamada J, Yano S, Kai Yet al.Histopathological study of venous aneurysms in patients with dural arteriovenous fistulas. J Neurosurg 2000;92(06):1023-1027

30 Awad IA, Little JR, Akarawi WP, Ahl J. Intracranial dural arteriovenous malformations: factors predisposing to an aggressive neurological course. J Neurosurg 1990;72(06):839-850

31 Lucas CdeP, Caldas JG, Prandini MN. Do leptomeningeal venous drainage and dysplastic venous dilation predict hemorrhage in dural arteriovenous fistula? Surg Neurol 2006;66(Suppl 3):S2-S5

32 Houser OW, Baker HLJr, Rhoton AL Jr, Okazaki H. Intracranial dural arteriovenous malformations. Radiology 1972;105(01):55-64

33 Bulters DO, Mathad N, Culliford D, Millar J, Sparrow OC. The natural history of cranial dural arteriovenous fistulae with cortical venous reflux-the significance of venous ectasia. Neurosurgery 2012;70(02):312-318

34 Grisoli F, Vincentelli F, Fuchs Set al.Surgical treatment of tentorial arteriovenous malformations draining into the subarachnoid space. Report of four cases. J Neurosurg 1984;60(05):1059-1066

35 Halbach VV, Higashida RT, Hieshima GB, Mehringer CM, Hardin $\mathrm{CW}$. Transvenous embolization of dural fistulas involving the transverse and sigmoid sinuses. AJNR Am J Neuroradiol 1989; 10(02):385-392

36 King WA, Martin NA. Intracerebral hemorrhage due to dural arteriovenous malformations and fistulae. Neurosurg Clin N Am 1992;3(03):577-590

37 Duffau H, Lopes M, Janosevic Vet al.Early rebleeding from intracranial dural arteriovenous fistulas: report of 20 cases and review of the literature. J Neurosurg 1999;90(01):78-84 
130 Superior Sagittal Sinus Wall Fistula with Direct Cortical Venous Drainage Chavan et al.

38 Thompson BG, Doppman JL, Oldfield EH. Treatment of cranial dural arteriovenous fistulae by interruption of leptomeningeal venous drainage. J Neurosurg 1994;80(04):617-623

39 Collice M, D’Aliberti G, Talamonti Get al.Surgical interruption of leptomeningeal drainage as treatment for intracranial dural arteriovenous fistulas without dural sinus drainage. J Neurosurg 1996;84(05):810-817
40 Chandler HC Jr, Friedman WA. Successful radiosurgical treatment of a dural arteriovenous malformation: case report. Neurosurgery 1993;33(01):139-141

41 Guo WY, Pan DH, Wu HMet al.Radiosurgery as a treatment alternative for dural arteriovenous fistulas of the cavernous sinus. AJNR Am J Neuroradiol 1998;19(06):1081-1087 